\title{
The dwarf galaxy population in two compact groups
}

\author{
Patricia E. Campos ${ }^{1} \dagger$, C. Mendes de Oliveira ${ }^{1}$ \\ and M. Bolte ${ }^{2}$ \\ ${ }^{1}$ Instituto de Astronomia, Geofísica e Ciências Atmosféricas, Departamento de Astronomia, \\ Universidade de São Paulo, Brazil email: campos@astro.iag.usp.br \\ ${ }^{2}$ UCSC/Lick Observatory
}

\begin{abstract}
We have undertaken an imaging survey of low surface brightness dwarf galaxies in nearby groups with the main goal of studying the faint-end of the galaxy luminosity function at $\mathrm{z} \sim 0$. Here we describe the results on the search for LSB dwarf galaxies in two compact groups: HCG44 and HCG68. Our study explores the surface brightness, sizes, magnitudes and colours of these candidate dwarf galaxies. The selection criteria were their central surface brightness, scale factors and diameters at the limiting isophote of $26 \mathrm{R} \mathrm{mag} /{ }^{\prime 2}$. We estimate the faint end of the luminosity function of galaxies through Monte Carlo simulations. The observed magnitude distribution of the candidate dwarf galaxies in the groups (down to $M_{R} \sim-13$ ) is compatible with a Schechter function with a slope $\alpha \sim-1.2$.
\end{abstract}

\section{Observations, selection criteria and analysis}

We have obtained CCD images which cover $1.18 \mathrm{deg}^{2}$ and $0.77 \mathrm{deg}^{2}$ of the central regions of the groups HCG44 and HCG68, respectively, with the KPNO 0.9m telescope in the B and R bands. HCG44 has 4 bright galaxies (3 late-type and 1 early-type) and HCG68 has 5 bright members (4 early-type and 1 late-type). We adopt a distance of 34 $\mathrm{Mpc}$ to both groups. Detection and photometry of sources were done with the program Source Extractor (Bertin \& Arnouts 1996). The selection criteria were based on the properties of the Local Group dwarf galaxies, projected to the group's distance, as in Carrasco et al. (2001). We have selected objects with $\mu_{0}>21 \mathrm{R} \mathrm{mag} /{ }^{2}$, scale factor $h>2$ " and diameters $>6$ ". We assumed that the dwarf galaxies have exponential profiles (Ferguson \& Binggeli 1994). All objects were visually inspected. We have selected a total of 35 and 45 objects from HCG44 fields and HCG68 fields, respectively. Figure 1 shows a mosaic of their images.

\section{Colour distribution}

HCG44 has only 18 galaxies with measured colours and the group HCG68 has 37 galaxies. The group HCG68 has a more pronounced peak at $B-R \sim 1.1$ while HCG44 has a more diffuse distribution, with two pronounced peaks, one at $B-R \sim 0.9$ and the other at $B-R \sim 2.5$. The red peak is probably due to contaminating background galaxies. It was observed that there are differences in the population of dwarf galaxies in HCG68 and HCG44. The group HCG68 is richer in dwarf galaxies and has a more concentrated colour distribution. This may reflect the fact that HCG68 is in a denser environment than HCG44. HCG68 is early-type dominated and may be a substructure

$\dagger$ Present address: IAG-USP, Departamento de Astronomia, Rua do Matão 1226, Cidade Universitária 05508-900, São Paulo, SP, Brazil. 

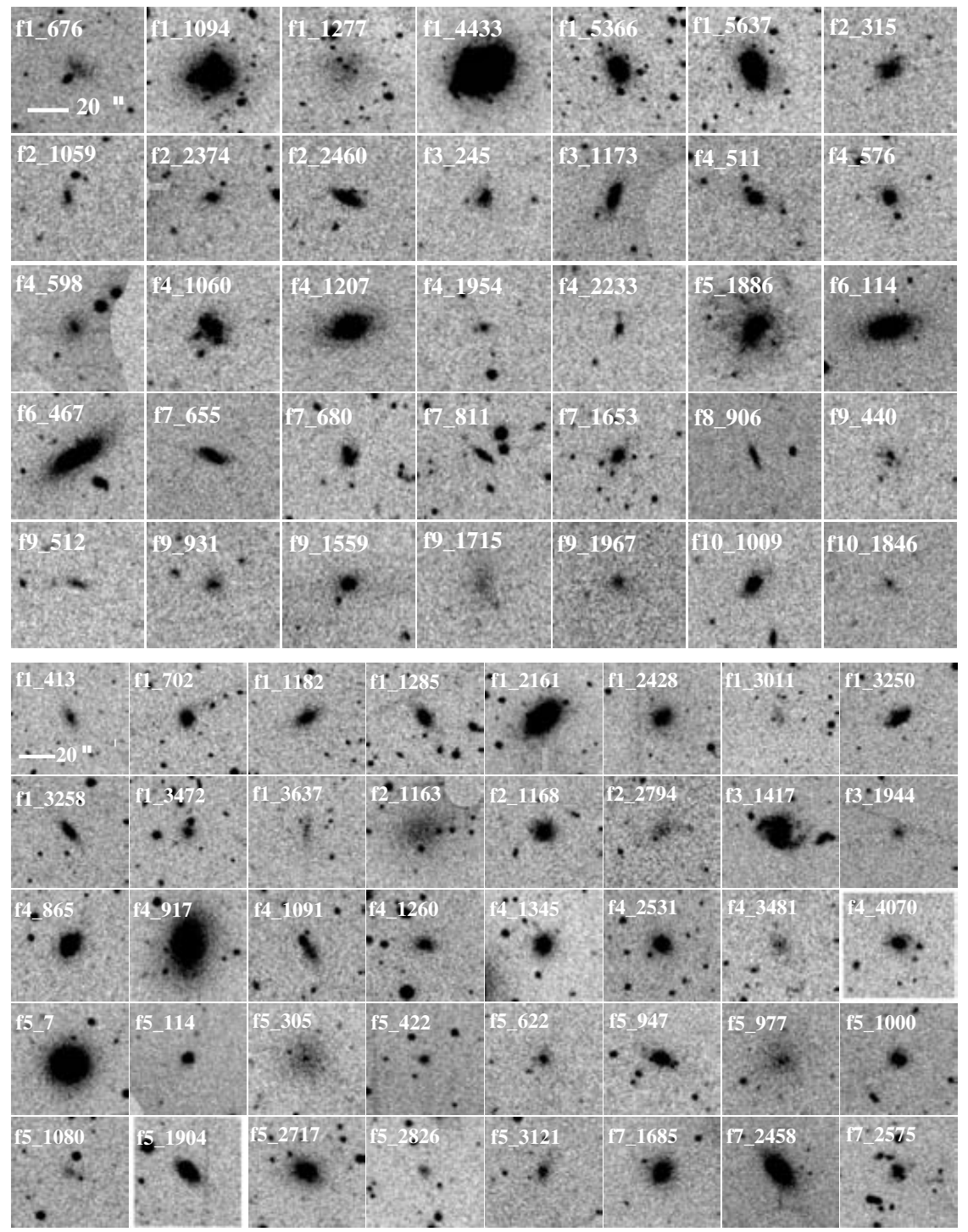

Figure 1. Mosaic of the candidate dwarf galaxies of the groups HCG44 (top) and HCG68 (bottom).

inside a loose group. One of the major problems when studying the faint dwarf galaxy population in groups or clusters is to distinguish group members from giant background galaxies. Figure 2 shows the scale-factor $h$ vs the central surface brightness $\mu_{0}$ for the candidate dwarf galaxies. One can readily see the reddest galaxies of the sample are also those with the highest central surface brightness, and these are most probably galaxies in the background.

\section{Monte Carlo simulations: the faint-end of the LF}

In this section we use Monte-Carlo simulations to test the Schechter function parameters which best fit the observed magnitude distribution of the galaxies. For that, we 


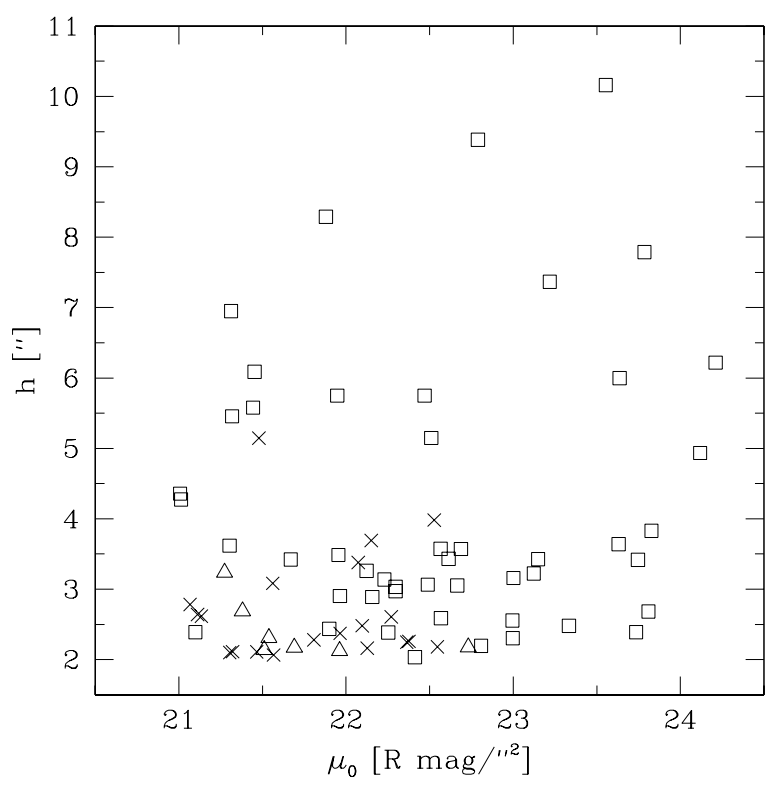

Figure 2. Scale-factor vs central surface brightness for the candidate dwarf galaxies in both groups. The squares represent colours $B-R \leqslant 1.7$ and the triangles represent the $B-R>1.7$. Crosses indicates the galaxies with no colour information.

simulate 4000 galaxies for each $M^{*}$ and $\alpha$ pair tested, and then we perform the same process to detect and measure the objects, as done for the real galaxies. The magnitude distribution of the recovered galaxies were then compared with the magnitude distribution of the real galaxies through a Kolmogorov-Smirnov (K-S) test. In addition, a $\chi^{2}$ test on the binned data was performed in order to confirm the results. The observed magnitude distribution of the candidate dwarf galaxies in the groups (down to $M_{R} \sim-13$ ) is compatible with a Schechter function with a slope $\alpha \sim-1.2$. We find that both HCG44 and HCG68 have luminosity functions similar to that seen in the Local Group (van den Bergh 2000), without the enhanced numbers of dwarf galaxies observed in larger groups like Virgo or Fornax. Thus, our results are in agreement with the observed trend of a flatter slope of the faint end of the luminosity function for groups $\sim-1.2$ (Trentham \& Tully 2002, Flint et al. 2001) than predicted from CDM models.

\section{Acknowledgements}

P.E. Campos acknowledges the financial support from IAU, PRPG-USP and CNPQ. $\mathrm{CMdO}$ acknowledges funding from the Alexander von Humboldt Foundation.

\section{References}

Bertin, E. \& Arnouts, S. 1996, A\&AS, 117, 393

Carrasco, E. R., Mendes de Oliveira, C., Infante, L. \& Bolte, M. 2001, AJ, 121, 148

Ferguson, H. C., \& Binggeli, B., 1994, A\&ARv, 6, 67

Flint K., Metevier, A. J., Bolte, M. \& Mendes de Oliveira, C. 2001, ApJS, 134, 53

Trentham, N., Tully, R.B., 2002, MNRAS, 335, 712

van den Bergh, S., 2000, PASP, 112, 529 